\title{
Mechanical touch responses of Arabidopsis TCH1-3 mutant roots on inclined hard-agar surface**
}

\author{
Guodong Zha, Bochu Wang*, Junyu Liu, Jie Yan, Liqing Zhu, and Xingyan Yang \\ Key Laboratory of Biorheological Science and Technology, Ministry of Education, College of Bioengineering, \\ Chongqing University, Chongqing 400044, China
}

Received April 20, 2015; accepted October 7, 2015

\begin{abstract}
A b s t r a c t. The gravity-induced mechanical touch stimulus can affect plant root architecture. Mechanical touch responses of plant roots are an important aspect of plant root growth and development. Previous studies have reported that Arabidopsis TCH1-3 genes are involved in mechano-related events, however, the physiological functions of TCH1-3 genes in Arabidopsis root mechanoresponses remain unclear. In the present study, we applied an inclined hard agar plate method to produce mechanical touch stimulus, and provided evidence that altered mechanical environment could influence root growth. Furthermore, tch1-3 Arabidopsis mutants were investigated on inclined agar surfaces to explore the functions of TCH1-3 genes on Arabidopsis root mechanoresponses. The results showed that two tch 2 mutants, cml24-2 and $c m l 24-4$, exhibited significantly reduced root length, biased skewing, and decreased density of lateral root. In addition, primary root length and density of lateral root of $t \mathrm{ch} 3(\mathrm{cml12}-2)$ was significantly decreased on inclined agar surfaces. This study indicates that the $t \operatorname{ch} 2$ and $t \operatorname{ch} 3$ mutants are hypersensitive to mechanical touch stimulus, and TCH2 (CML24-2 and CML24-4) and TCH3 (CML12-2) genes may participate in the mechanical touch response of Arabidopsis roots.

$\mathrm{K}$ e y w o r d s: mechanical touch stimulation, TCH1-3 genes, root growth, Arabidopsis
\end{abstract}

\section{INTRODUCTION}

Mechanical stimulus experienced by roots induces continuous root response as roots are growing in soil (Gleeson et al., 2012). In natural environment, the main sources of mechanical stimulation for plants are wind, touch, rain and obstacle (Coutand, 2010). Higher plant roots change their growth patterns in response to these mechanical stimuli (Okada et al., 1990). When root tips encounter obstacles in soil, they avoid the obstacles by changing the direction

*Corresponding author e-mail: wangbc2000@126.com **This work was supported by the fund of National Natural Science Foundation of China (No. 11172337), 2012-2015. of their growth (Monshausen and Gilroy, 2009b). Besides, the positive gravitropism and touch stimuli can affect many growth patterns of plant roots on the surface of agar, such as waving, skewing, helix and circumnutation (Chehab et al., 2009; Migliaccio et al., 2013; Okada and Shimura, 1990; Silverberg et al., 2012). Therefore, exploring the cellular and molecular basis of roots mechanoresponses is of great importance for fundamental plant biology and agricultural practice.

Gravity-induced touch stimulus can give rise to two characteristic root growth patterns of Arabidopsis, waving and skewing, when Arabidopsis seedlings grow on impenetrable and inclined agar surface (Okada and Shimura, 1990; Rutherford and Masson, 1996). This altered growth behaviour is proposed to be a touch-induced resistance based on gravity perception or response and may enable the fine navigation ability of plant roots during growth through soil (Massa and Gilroy, 2003). In addition, root branching can also be affected by mechanical forces (Richter et al., 2009). Previous studies have reported that lateral roots emerge from the convex side of a curved root, whether the curvature is formed by waving or physical bending (Ditengou et al., 2008; Richter et al., 2009). Despite these many mechanical-induced root growth behaviours, the molecular mechanisms of mechanoresponse remain largely unknown (Monshausen and Gilroy, 2009a,b).

In order to explore the molecular mechanisms of mechanoresponses in Arabidopsis root, Braam and Davis (1990) isolated four touch-induced TCHs (touch inducible genes). Among the four genes, TCH1-3 genes encode Calmodulin (CaM) and Calmodulin-like (CML)

(C) 2016 Institute of Agrophysics, Polish Academy of Sciences 
proteins, which suggests potential involvement of $\mathrm{Ca}^{2+}$ and potential $\mathrm{Ca}^{2+}$ receptors in Arabidopsis mechanoresponses (Braam and Davis, 1990). Although recent reports implicate intracellular $\mathrm{Ca}^{2+}$ as the second message in plant mechano-transduction pathways leading to appropriate mechanoresponses, such as primary root elongation and lateral root initiation (Monshausen et al., 2009; Richter et al., 2009), mechano-related functions of TCH1-3 genes have not been investigated.

The effects of numerous genes on mechanoresponses of roots have been demonstrated (Braam, 1992; Braam and Davis, 1990; Lee et al., 2005), however, the functions of TCH1-3 in the mechanoresponses are still unclear. Thus, in the present work, we took advantage of root length, skewing degree, and the density of lateral root (DLR) assays to explore the potential mechanoresponses functions of TCH1-3 genes in Arabidopsis root behaviours using a reverse genetic approach. We found that the tch1-3 mutants roots tested showed distinct phenotypic consequences on inclined agar surface. This study indicated that $t c h 2$ and tch3 mutants were hypersensitive to increased levels of mechanical stimuli, namely the normal function of the TCH2 (CML24-2 and CML24-4) and TCH3 (CML12-2) might be necessary for Arabidopsis primary root in response to mechanical touch stimulus.

\section{MATERIAL AND METHOD}

The wild-type (WT) tch 1 (cam2-1 and cam2-3), tch2 (cml24-2 and cml24-4) and tch3 (cml12-2) Arabidopsis mutants were used in this study (Tsai et al., 2007). Arabidopsis seeds were surface-sterilised with $75 \%$ ethanol for $1 \mathrm{~min}$ and half-strength bleach for $10 \mathrm{~min}$. Sterilized seeds were cold-treated at $4^{\circ} \mathrm{C}$ for 2 days before being sown on a growth medium containing half-strength Murashige and Skoog and $1.5 \%(\mathrm{w} / \mathrm{v})$ sucrose, and solidified with $1.2 \%(\mathrm{w} / \mathrm{v})$ agar (Sigma, product No. A4675), $\mathrm{pH}$ 5.8. The plates were sealed with micropore tape and grown at $22^{\circ} \mathrm{C}$ with a $16 \mathrm{~h}$ light $/ 8 \mathrm{~h}$ dark cycle before imaging.

In the present study, mechanical touch stimuli were performed as previously described (Okada and Shimura, 1990). The 2 days cold-treated Arabidopsis seeds were sown on the agar surface of the growth medium (each plate with 8 seeds in a row). The plates were placed in racks where they were maintained at $0,15,30,45$, and $60^{\circ}$ angles from the gravity vector (Fig. 2A), and then incubated at $22^{\circ} \mathrm{C}$ with a $16 \mathrm{~h} \mathrm{light} / 8 \mathrm{~h}$ dark cycle before imaging.

On inclined agar plates, the hard agar surface presents a barrier that mechanically impedes the root, while at the same time downward growth in response to gravity causes the root tip to continuously push against the agar surface. The resulting mechanical cues influence the primary root growth and development and primary root tip direction, as well as the lateral root formation. To test the mechanoresponse behaviour of $t c h 1 \sim 3$ mutants under mechanical stimuli, we took the average length and skewing angle of primary roots and lateral root density into consideration. The primary root length and root skewing were measured after 7 days of cultivation. Photographic images were taken above each plate with an Olympus FE-320 digital camera. Primary root length $(\mathrm{mm})$ was defined as the actual length starting from the shoot-root junction to the root tip. Root skewing degree was defined as the angle between the gravitational vector and the growth vector starting from the shoot-root junction to the root tip. The positive value was referred to left skewing, while the negative value was referred to right skewing. Density of lateral root (No./mm) was customarily calculated as the ratio between the number of lateral roots and the total length of the primary root after 12 days of cultivation.

Projections of microscopy and all digital measurements were performed with Image J 1.46 software (NIH, USA), and all statistical analyses were performed by IBM $^{\circledR}$ SPSS ${ }^{\circledR}$ Statistics software (IBM, Armonk, NY, USA), and all figures were plotted with Origin 8.5.1 (Origin-Lab Corporation, USA). Statistical analysis was carried out using Student $t$ test.

\section{RESULTS}

In our study, to explore the effects of mechanical touch stimulus on root growth and development, we had to establish an effective mechanical stimulus approach. To this end we chose to cultivate wild-type Arabidopsis seeds on $15,30,45$, and $60^{\circ}$ inclined and vertically positioned agar plates. Roots growing along the surface of agar were mechanically stimulated by contact between the root tip and the agar surface, and the level of mechanical touch stimulus could increase with the plate angle (Okada and Shimura, 1990; Thompson and Holbrook, 2004). Quantitative analysis showed that mechanical touch stimulus significantly stimulated primary roots elongation at $30^{\circ}$ from the vertical (Fig. 1A). However, the high level of touch stimulation $\left(30^{\circ}<\theta<60^{\circ}\right)$ would inhibit primary roots elongation. Furthermore, as seen in Fig. 1B, mechanical touch stimulus could promote lateral root growth and development, and the DLR was significantly increased at $30^{\circ}$ from the vertical. As the characteristic root growth pattern on the surface of agar plate, the skewing growth pattern was reported by numerous studies (Monshausen and Gilroy, 2009b; Oliva and Dunand, 2007; Qi and Zheng, 2013; Wang et al., 2011). In our experiments, the results showed that primary roots exhibited slight, insignificant left skewing at $30^{\circ}$ from the vertical (Figs $1 \mathrm{C}$ and $3 \mathrm{~A}$ ), indicating that the roots growth direction was along the gravitational vector on inclined plates. Those results show that cultivating seedlings on inclined agar surface is sufficient to develop a mechanical touch stimulus, and at $30^{\circ}$ inclined condition the mechanical force was more capable to affect both the primary root elongation and direction and 

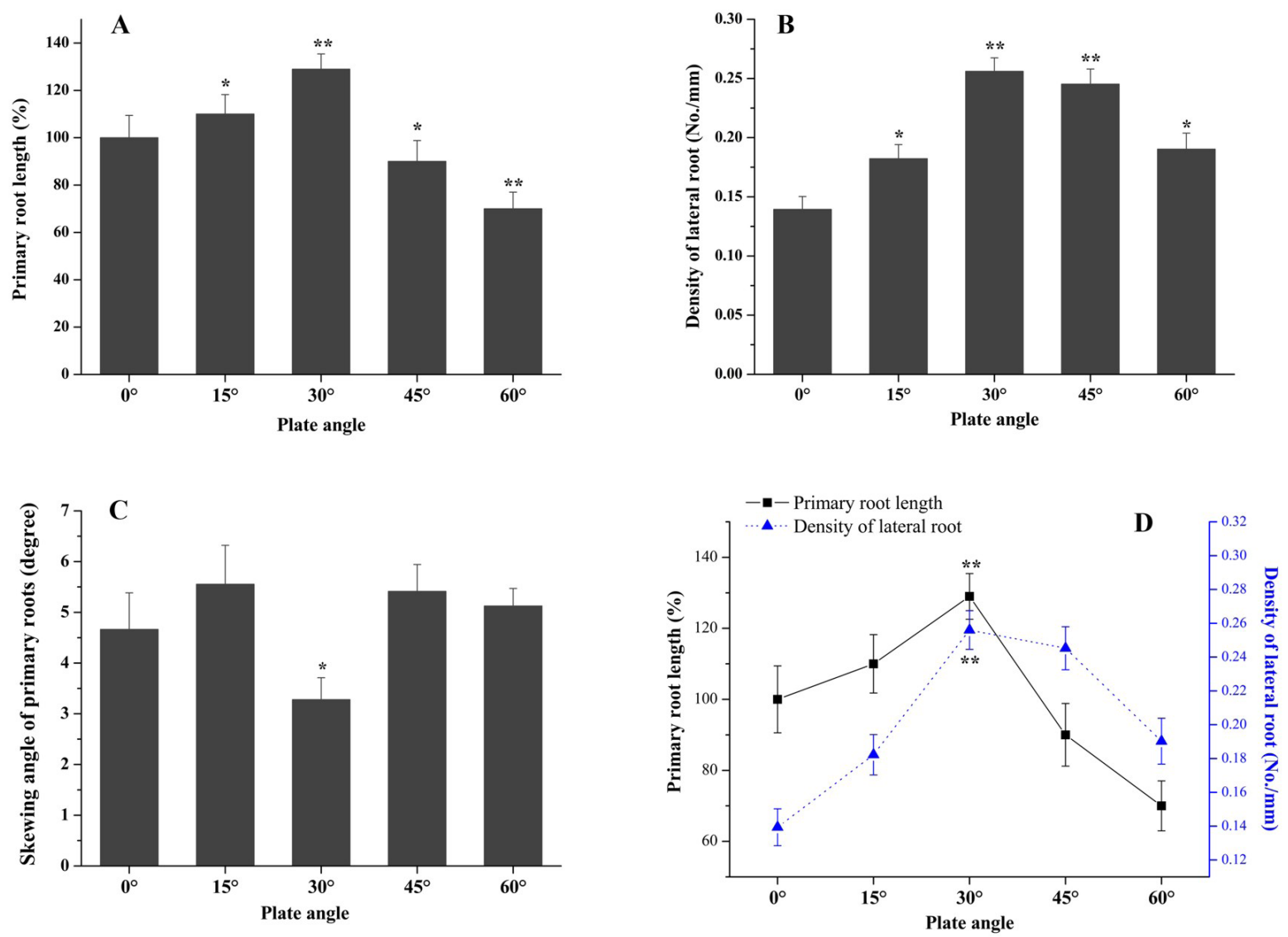

Fig. 1. The wild-type roots response to various angles of hard agar surface $\left(0,15,30,45\right.$, and $60^{\circ}$ from the vertical). $A-$ average length of primary root, $\mathrm{B}$ - density of lateral root, $\mathrm{C}$-skewing angle of primary root, $\mathrm{D}$ - both primary roots and lateral roots exhibit significant discrepancies at $30^{\circ}$ from the vertical compared to the other groups. Data are means $\pm \mathrm{SD}(\mathrm{n}>40) ;{ }^{*} \mathrm{p}<0.05,{ }^{*} \mathrm{p}<0.01$, t test.

the lateral root formation. Taken together, we selected the $30^{\circ}$ from the vertical cultivating environment for subsequent experiments.

According to the results above, we found that the effects of touch stimulus on root growth and development were different, and significant discrepancies were exhibited at $30^{\circ}$ from the vertical (Fig. 1D). Therefore, to assess tch 1 (cam2-1 and cam2-3), tch2 (cml24-2 and cml24-4), and $t c h 3(c m l 12-2)$ responses to mechanical touch stimulation, root growth was analyzed in seedlings growing at $30^{\circ}$ from the vertical. When grown on inclined plates, tch1-3 mutant roots displayed indistinguishable waving patterns from those of WT (Fig. 3), but they were apparent (Okada and Shimura, 1990). Quantitative analysis revealed that primary root length of $t c h 1$ (cam2-1 and cam2-3) mutants was significantly longer $(\mathrm{p}<0.05)$ than that of WT (Fig. 2B). However, the primary roots elongation of $t c h 2$ (cml24-2 and cml24-4) and tch3 (cml12-2) was significantly inhibited $(\mathrm{p}<0.05$ or 0.01$)$ on the inclined agar plates
(Fig. 2B). These suggested that CML24 and CML12 were involved in promoting primary root elongation in response to mechanical stimulus.

In addition, the root skewing degree of tch1-3 Arabidopsis primary roots exhibited the left- or right-skewing phenotypes in response to mechanical touch stimulus (Figs $2 \mathrm{C}$ and 3). However, two functionally disrupted points mutant lines for $t c h 2, c m l 24-2$ and cml24-4 exhibited significantly right- and left-skewing behaviour relative to that of WT, respectively (Figs 2C, 3E, 3F). In our experiments, all the primary roots presented the waving growth pattern when growing on inclined agar plates, while primary roots of $t c h 2$ mutants appeared to skew towards one side, suggesting that $C M L 24$ and $C M L 12$ inhibited primary root growth direction under mechanical stimuli.

The lateral root is one of the most important organs for plant growth and development. The DLR is widely used in current literature, representing an estimation of overall biomass of the root system (De Smet et al., 2012; Dubrovsky et al., 2006). To explore the effects of mechanical touch stimulus on lateral root growth and development of tch1-3 
A

\section{Schematic diagram of touch stimulation}
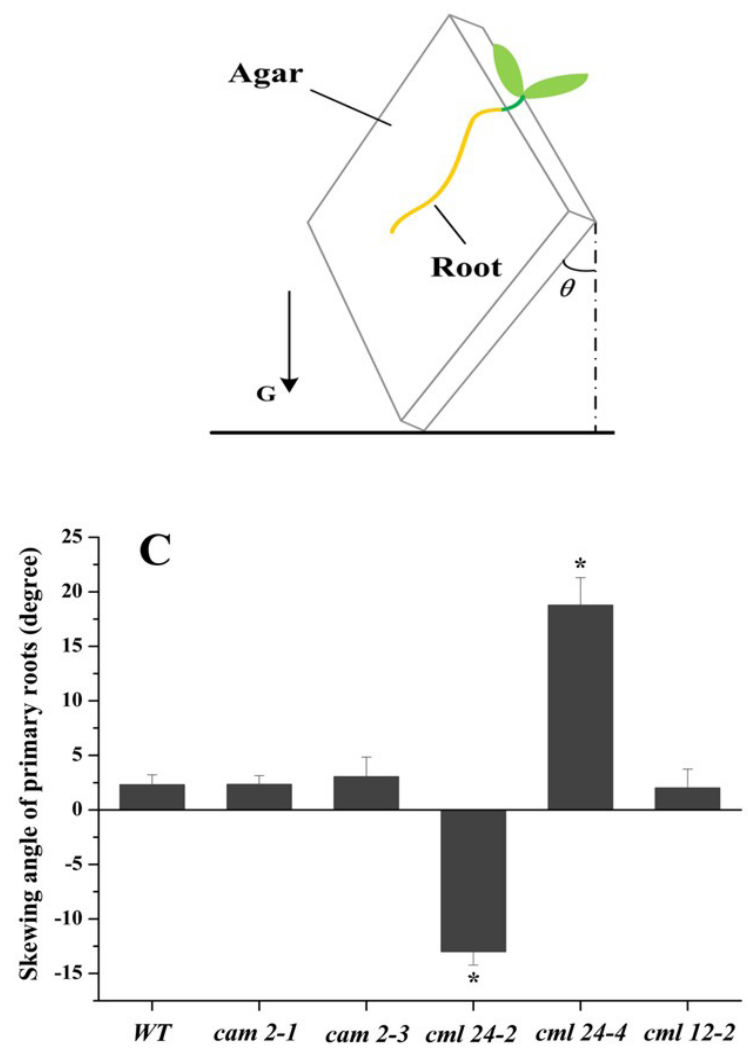

B
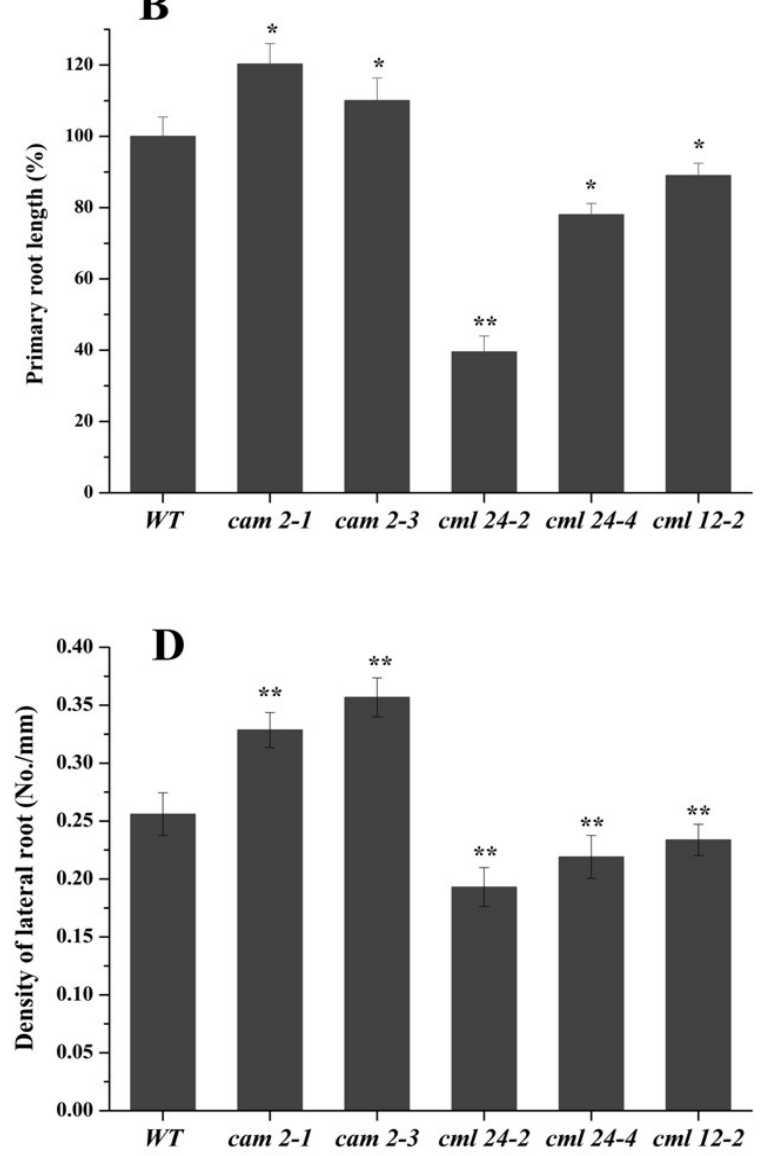

Fig. 2. Effects of mechanical touch stimulus on root growth of tch1-3 mutants. A - schematic diagram of touch stimulation; plates maintained at $0,15,30,45$, and $60^{\circ}$ angles $(\theta)$ between the root growth axis and the gravity vector; $\mathrm{B}, \mathrm{C}$, and $\mathrm{D}-$ represent primary root length, root skewing and DLR of $t c h 1-3$ and WT on inclined plates, respectively. In panel (C), positive values refer to left skewing, while negative values refer to right skewing. Explanations as in Fig. 1.

mutants, we analyzed the DLR of all mutants after 12-day cultivation. Our results showed that mechanical touch stimulus could increase the DLR of WT at $30^{\circ}$ from the vertical (Fig. 1B). However, quantitative analysis showed that the DLR of $t c h 2(\mathrm{cml} / 24-2$ and $\mathrm{cm} / 24-4)$ and $t \mathrm{ch} 3(\mathrm{cml}$ 12-2) were significantly $(\mathrm{p}<0.01)$ decreased (Fig. 2D) on inclined plates $\left(30^{\circ}\right)$, indicating that $C M L 24$ and $C M L 12$ were involved in mechano-touch responses of lateral roots. Remarkably, the DLR of tch 1 (cam2-1 and cam2-3) was significantly $(\mathrm{p}<0.01)$ increased at $30^{\circ}$ from the vertical (Fig. 2D), so it was possible that the TCH1 gene defect had no effect on mechanical stimulation of lateral root formation, or it suppressed periodically lateral root formation to some extent; all these need further verification.

\section{DISCUSSION}

Although more and more reports demonstrate the fundamental function of mechanostimulus in the regulation of plant growth and development, the molecular basis for plant mechanoresponses is still largely unknown (Chehab and Eich, 2009; Coutand, 2010; Monshausen et al., 2009; Monshausen and Gilroy, 2009a; Telewski, 2006). In the present study, we investigated the touch mechanoresponses of Arabidopsis roots, and found that the touch stimulus could affect both the primary root and lateral root growth behaviour. To further explore the effect of touch stimulus on regulating root growth, tch1-3 Arabidopsis mutants were used to reveal the potential functions of $T C H 1-3$ genes in Arabidopsis root mechanoresponses by reverse genetic approach (Tamura et al., 2010). In our mechanical stimulation model, the force acting on roots is a complex resultant, the force arising from the contact between the root tip and the agar surface being beyond quantification. In our experiments we just selected a fixed inclined angle $\left(30^{\circ}\right.$ from the vertical), which would develop a sustainable and stable resultant force on roots. Under this condition, we discussed the function of $T C H 1 \sim 3$ in response to mechanical stimuli. 

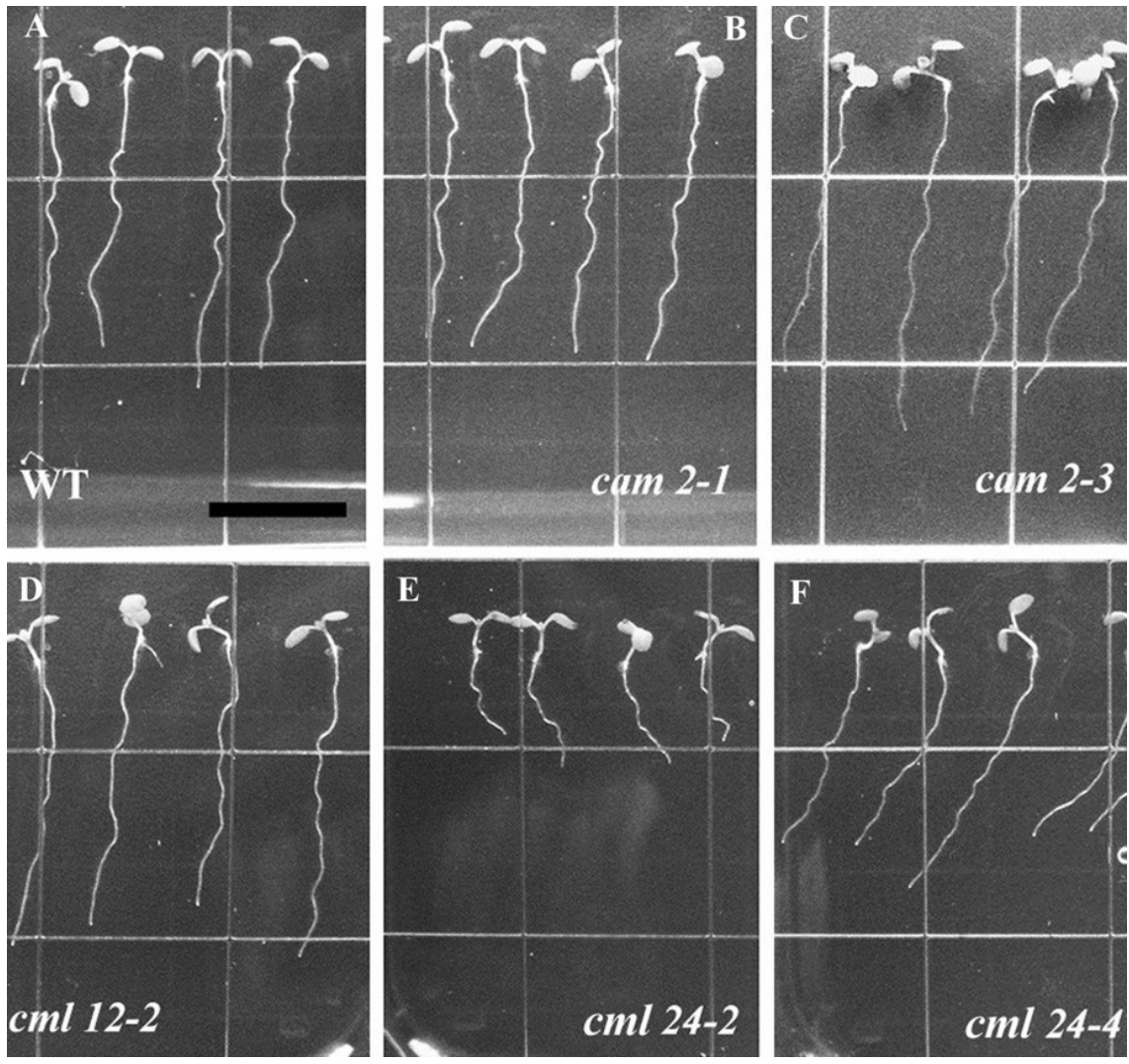

Fig. 3. Growth patterns of agar surface-grown primary roots of tch1-3 mutants and WT Arabidopsis. Examples of primary roots of 7-days-old WT (A), cam2-1 (B), cam2-3 (C), cml12-2 (D), cml24-2 (E) and cml24-4 (F) grown on agar surfaces. Note the similar waving growth of all the genotypes, and that $\mathrm{cm} / 24-2$ roots skew significantly to the right and $\mathrm{cm} / 24-4$ roots skew significantly to the left (Bar scale in $\mathrm{A}-\mathrm{F}=10 \mathrm{~mm}$ ).

Gravity-induced touch stimulus can result in primary root waving and skewing on hard agar surface (Oliva and Dunand, 2007). Although waving and skewing are two growth behaviours usually occurring at the same time, skewing can also be found without obvious waving (Yuen et al., 2005). Our observation also supports the uncoupling of these two behaviours (Fig. 3). All the mutants tested in this work exhibited similar waving behaviours to those of the WT. However, evidence supporting the conclusion that $t c h 2$ root response to mechanical cues comes from the analysis of mechanically stimulated roots growing on tilted agar plates and other mechanostimulus (Braam, 1992; Henry-Vian et al., 1995; Sistrunk et al., 1994; Wang et al., 2011). We have found that $t$ ch 2 roots growing on inclined plates appear left- and right skewed compared with those of WT (Fig. 2C, 3E, 3F). These results suggest that the activities of CML24-2 and CML24-4 could repress touch-induced root phenotypes. Furthermore, the increased repressions observed in $c m / 24-2$ and $c m l 24-4$ mutants are greater than that of WT, indicating that the tch 2 root is hypersensitive to an increase in mechanical stimulus, and the $t c h 2$ mutants roots make use of a positive regulatory pathway that activates skewing in response to touch stimu- lus. In other words, the activities of $C M L 24-2$ and $C M L$ 24-4 could regulate the Arabidopsis root growth behaviour in response to mechanical touch stimulus.

Noteworthy is the fact that the loss of CML24 and $C M L$ 12 activities has opposing effects on root length. In touch stimulated tch 2 and tch 3 mutant roots, average length of primary roots is shorter while skewing is enhanced, indicating that CML24 and CML12 activities promote the elongation and inhibit the skewing of primary roots responding to mechanical cues. This phenomenon could explain the opposing effects of CML24 and CML12 on touch-stimulus, and gravity-mediated growth is as follows: the proteins can reduce skewing and promote elongation indirectly, by reinforcing gravity responses when mechanically stimulated (Gleeson et al., 2012). Besides, from Fig. 2 we believe that the sensitivity of $C M L 12$ to mechanical stimulus is possibly lower than that of $C M L 24$, because the remarkable defective mechanoresponses behaviour of cml24 is more distinct than that of $c m l 12$. However, the specific mechanisms need further verification.

The lateral root could improve the capability of the root system to acquire nutrients and water. In addition, the DLR is one of the most important indexes to evaluate the lateral root development (De Smet et al., 2012; Dubrovsky et al., 
2006). Previous studies have reported that the lateral root is conducive to plant response to mechanostimulus, especially the mechanical bending (Ditengou et al., 2008; Hamant et al., 2008; Richter et al., 2009). In our experiments, the appropriate mechanical touch stimuli (at 30 and $45^{\circ}$ from the vertical) could significantly stimulate $(p<0.01)$ the lateral root development of WT (Fig. 1B), which was consistent with that mentioned above. Moreover, the DLR of $c m l 24$ and $c m l 12$ are lower than those of the others (Fig. 2D), indicating that CML24 and CML12 are involved in mechano-touch responses of lateral roots.

Although we did not discover the significant defective mechanoresponses behaviour of $t c h 1$ in our work, the functional redundancy of CAMs and CMLs family may remedy the defective function of the deficiency of TCH1 (Delk et al., 2005; McCormack and Braam, 2003; McCormack et al., 2005). Taken together, the normal function of the TCH 1-3 genes may be necessary for Arabidopsis primary root mechanoresponse in the present study, especially $\mathrm{TCH} 2$ (CML24-2 and CML24-4) and TCH3 (CML12-2), and the potential roles of $C A M s$ and $C M L s$ in mechano-related physiological events will also be an important future research direction.

\section{CONCLUSIONS}

1. The $30^{\circ}$ from the vertical cultivating condition is the most favourable and efficient to produce a sustainable and stable mechanical touch stimulus in the growing root system of Arabidopsis.

2. TCH2 (CML24-2 and CML24-4) and TCH3 (CML 12-2) genes are involved in the response to mechanical stimuli in Arabidopsis root system, while the role of TCH1 in root system mechanoresponses seems to be not important, which still needs further exploration.

\section{REFERENCES}

Braam J., 1992. Regulated expression of the calmodulin-related TCH genes in cultured Arabidopsis cells: induction by calcium and heat shock. Proc. National Academy of Sciences, 89(8), 3213-3216.

Braam J. and Davis R.W., 1990. Rain-induced, wind-induced, and touch-induced expression of calmodulin and calmodulin-related genes in arabidopsis. Cell, 60(3), 357-364.

Chehab E.W., Eich E., and Braam J., 2009. Thigmomorphogenesis: a complex plant response to mechano-stimulation. J. Experimental Botany, 60(1), 43-56.

Coutand C., 2010. Mechanosensing and thigmomorphogenesis, a physiological and biomechanical point of view. Plant Sci., 179(3), 168-182.

De Smet I., White P.J., Bengough A.G., Dupuy L., Parizot B., Casimiro I., Heidstra R., Laskowski M., Lepetit M., Hochholdinger F., Draye X., Zhang H., Broadley M.R., Peret B., Hammond J.P., Fukaki H., Mooney S., Lynch J.P., Nacry P., Schurr U., Laplaze L., Benfey P., Beeckman T., and Bennett M., 2012. Analyzing lateral root development: how to move forward. Plant Cell, 24(1), 15-20.
Delk N.A., Johnson K.A., Chowdhury N.I., and Braam J., 2005. CML24, regulated in expression by diverse stimuli, encodes a potential $\mathrm{Ca} 2+$ sensor that functions in responses to abscisic acid, daylength, and ion stress. Plant Physiology, 139(1), 240-253.

Ditengou F. A., Tealea W.D., Kochersperger P., Flittner K.A., Kneuper I., van der Graaff E., Nziengui H., Pinosa F., Li X., Nitschke R., Laux T., and Palme K., 2008. Mechanical induction of lateral root initiation in Arabidopsis thaliana. Proc. Nat. Acad. Sci. USA, 105(48), 18818-18823.

Dubrovsky J., Gambetta G., Hernandez-Barrera A., Shishkova S., and Gonzalez I., 2006. Lateral root initiation in Arabidopsis: developmental window, spatial patterning, density and predictability. Annals Botany, 97(5), 903-915.

Gleeson L., Squires S., and Bisgrove S.R., 2012. The microtubule associated protein END BINDING 1 represses root responses to mechanical cues. Plant Sci., 187, 1-9.

Hamant O., Heisler M.G., Jonsson H., Krupinski P., Uyttewaal M., Bokov P., Corson F., Sahlin P., Boudaoud A., Meyerowitz E.M., Couder Y., and Traas J., 2008. Developmental patterning by mechanical signals in Arabidopsis. Science, 322(5908), 1650-1655.

Henry-Vian C., Vian A., Davies E., Ledoigt G., and Desbiez M.O., 1995. Wounding regulates polysomal incorporation of hsp 70 and tch 1 transcripts during signal storage and retrieval. Physiologia Plantarum, 95(3), 387-392.

Lee D., Polisensky D.H., and Braam J., 2005. Genome-wide identification of touch- and darkness-regulated Arabidopsis genes: a focus on calmodulin-like and XTH genes. New Phytologist, 165(2), 429-444.

Massa G.D. and Gilroy S., 2003. Touch modulates gravity sensing to regulate the growth of primary roots of Arabidopsis thaliana. Plant J., 33(3), 435-445.

McCormack E. and Braam J., 2003. Calmodulins and related potential calcium sensors of Arabidopsis. New Phytologist, 159(3), 585-598.

McCormack E., Tsai Y.C., and Braam J., 2005. Handling calcium signaling: Arabidopsis CaMs and CMLs. Trends Plant Sci., 10(8), 383-389.

Migliaccio F., Tassone P., and Fortunati A., 2013. Circumnutation as an autonomous root movement in plants. Am. J. Bot., 100(1), 4-13.

Monshausen G.B., Bibikova T.N., Weisenseel M.H., and Gilroy S., 2009. $\mathrm{Ca}^{2+}$ regulates reactive oxygen species production and ph during mechanosensing in Arabidopsis roots. Plant Cell, 21(8), 2341-2356.

Monshausen G.B. and Gilroy S., 2009a. The exploring root root growth responses to local environmental conditions. Current Opinion Plant Biology, 12(6), 766-772.

Monshausen G.B. and Gilroy S., 2009b. Feeling green: mechanosensing in plants. Trends in Cell Biol., 19(5), 228-235.

Okada K. and Shimura Y., 1990. Reversible root-tip rotation in Arabidopsis seedlings induced by obstacle-touching stimulus. Science, 250(4978), 274-276.

Oliva M. and Dunand C., 2007. Waving and skewing: how gravity and the surface of growth media affect root development in Arabidopsis. New Phytologist, 176(1), 37-43.

Qi B. and Zheng H., 2013. Modulation of root-skewing responses by KNAT1 in Arabidopsis thaliana. The Plant J., 76(3), 380-392. 
Richter G.L., Monshausen G.B., Krol A., and Gilroy S., 2009. Mechanical stimuli modulate lateral root organogenesis. Plant Physiology, 151(4), 1855-1866.

Rutherford R. and Masson P.H., 1996. Arabidopsis thatiana sku mutant seedlings show exaggerated surface-dependent alteration in root growth vector. Plant Physiology, 111(4), 987-998.

Silverberg J.L., Noar R.D., Packer M.S., Harrison M.J., Henley C.L., Cohen I., and Gerbode S.J., 2012. 3D imaging and mechanical modeling of helical buckling in Medicago truncatula plant roots. Proc. National Academy of Sciences, 109(42), 16794-16799.

Sistrunk M.L., Antosiewicz D.M., Purugganan M.M., and Braam J., 1994. Arabidopsis tch 3 encodes a novel $\mathrm{Ca}^{2+}$ binding-protein and shows environmentally-induced and tissue-specific regulation. Plant Cell, 6(11), 1553-1565.

Tamura W., Hidaka Y., Tabuchi M., Kojima S., Hayakawa T., Sato T., Obara M., Kojima M., Sakakibara H., and Yamaya T., 2010. Reverse genetics approach to characterize a function of NADH-glutamate synthase 1 in rice plants. Amino Acids, 39(4), 1003-1012.
Telewski F.W., 2006. A unified hypothesis of mechanoperception in plants. Am. J. Bot., 93(10), 1466-1476.

Thompson M.V. and Holbrook N.M., 2004. Root-gel interactions and the root waving behavior of Arabidopsis. Plant Physiology, 135(3), 1822-1837.

Tsai Y.-C., Delk N.A., Chowdhury N.I., and Braam J., 2007. Arabidopsis potential calcium sensors regulate nitric oxide levels and the transition to flowering. Plant Signaling Behavior, 2(6), 446-454.

Wang Y., Wang B., Gilroy S., Chehab E.W., and Braam J., 2011. CML24 is involved in root mechanoresponses and cortical microtubule orientation in Arabidopsis. J. Plant Growth Regulation, 30(4), 467-479.

Yuen C.Y.L., Sedbrook J.C., Perrin R.M., Carroll K.L., and Masson P.H., 2005. Loss-of-function mutations of ROOT HAIR DEFECTIVE3 suppress root waving, skewing, and epidermal cell file rotation in Arabidopsis. Plant Physiology, 138(2), 701-714. 